Jour. of Inverse and Ill-Posed Problems, 6, N5, (1998), 515-520.

\title{
ON THE THEORY OF REPRODUCING KERNEL HILBERT SPACES
}

\author{
A.G. RAMM
}

Department of Mathematics, Kansas State University, Manhattan, KS 66506-2602, USA

ramm@math.ksu.edu

\begin{abstract}
The inner product in RKHS is described in abstract form. Some of the results, published earlier, are discussed from a general point of view. In particular, the characterization of the range of linear integral transforms and inversion formulas, announced in the works of Saitoh, are analyzed.
\end{abstract}

\section{Introduction.}

The theory of reproducing kernels was developed in $[\mathrm{A}]$, [S]. A recent review of the theory is [Sa1,Sa2], where the reader can find many references.

The basic result in $[\mathrm{A}]$ is the existence and uniqueness of a reproducing kernel Hilbert space (RKHS) corresponding to any self-adjoint nonnegative-definite kernel $K(p, q), p, q \in E$, where $E$ is a measure space. Let $H$ be a Hilbert space of functions defined on $E$, and $H \subset L^{2}(E)$. Assume that $K(\cdot, q)$ and $K(p, \cdot)$ belong to $H$. Let us assume that the linear operator $K: H \rightarrow H$, with the kernel $K(p, q)$, is injective. It is defined on all of $H$ since $K(p, \cdot) \in H$ by the assumption. Define RKHS $H_{K}$ inner product by the formula

$$
(f, g)_{H_{K}}:=[f, g]:=\left(K^{-1} f, g\right)=\left(K^{-\frac{1}{2}} f, K^{-\frac{1}{2}} g\right),
$$

where $(f, g):=(f, g)_{L^{2}(E)}, K^{-1}$ is the operator inverse to $K: H \rightarrow H$, and

$$
K f:=\int_{E} K(p, q) f(q) d q .
$$

The injectivity assumption can be dropped, but then one has to consider $K$ on the factor space $H / N(K)$, where $N(K):=\{f: K f=0\}$ is the null-space of $K$.

In the literature (e.g. see [Sa1,2]) the inner product in RKHS was not defined explicitly by formula (1.1). The definition of the inner product in $H_{K}$, given in [A] (and presented in [Sa1, p.36]) is implicit and contains some limiting procedure which is not described explicitly. In particular, it is not clear over which sets of $p$ and $q$ the summation in formula (11) in [Sa1, p.36] is taken. In [A] such a summation is taken over a finite set of points $p \in E$ and $q \in E$. The finite sums $\sum_{p} X_{p} K(\cdot, p)$, used in [Sa1, p.36] do not form a complete Hilbert space $H_{K}$, and the completion procedure is not discussed in sufficient details in [Sa1]. Our definition (1.1) of the inner product in $H_{K}$ coincides with the definition in [Sa1, p.36, formula (11)] if one takes $f$ and $g$ in (1.1) to be finite linear combinations of the functions of the type $K(p, \cdot)$ and $K(\cdot, q)$.

The reproducing property of the kernel $K(p, q)$ can be stated as follows:

$$
[f(\cdot), K(\cdot, q)]=f(q),
$$

and this formula can be easily derived from the definition (1.1) of the inner product in $H_{K}$ :

$$
[f(\cdot), K(\cdot, q)]:=\left(K^{-1} f, K(\cdot, q)\right)=\left(f, K^{-1} K(\cdot, q)\right)=(f, I(\cdot, q))=f(q) .
$$

1991 Mathematics Subject Classification. 44A05, 46E20.

Key words and phrases. ill-posed problems, RKHS, reproducing kernels, range, inner products, integral transforms. 
Here we have used the selfadjointness of the operator $K^{-1}$, and the fact that the distributional kernel of the identity operator $I$ is $\delta(p-q)$, the delta function, which is well defined on RKHS because the value $f(p)$ for any $p \in E$ is a bounded linear functional in $H$ :

$$
|f(q)| \leq\|f\|\|K(\cdot, q)\|
$$

where $\|f\|:=[f, f]^{\frac{1}{2}}$ is the norm in $H_{K}$.

The basic results of this paper are:

1) representation of the inner product in $H_{K}$ by formula (1.1),

and

2) clarification of the conditions from [Sa1,2] under which the range of the general linear transform, defined by formula (2.1) below, is characterized and inversion formulas for this transform are obtained.

\section{Linear transforms and RKHS.}

Define

$$
f(p):=L F:=\int_{T} \overline{h(t, p)} F(t) d m(t),
$$

where $T \subset \mathbb{R}^{n}$ is some subset of $\mathbb{R}^{n}, d m(t)$ is a positive measure on $T$, and $h(t, p)$ is a function on $H_{0} \times H$, where $H_{0}:=L^{2}(T, d m(t))$. The linear operator $L: H_{0} \rightarrow H$ is injective if the set $\{h(t, p)\} \forall p \in E$ is total in $H_{0}$. This means that if for some $F \in L^{2}(T, d m(t))$ the following equation holds:

$$
0=\int_{T} h(t, p) F(t) d m(t) \quad \forall p \in E
$$

then $F(t)=0$.

Let us assume that $L$ is injective. The operator $L^{*}: H \rightarrow H_{0}$ acts by the formula:

$$
(L F, g)_{H}=\left(F, L^{*} g\right)_{H_{0}}
$$

thus

$$
L^{*} g=\int_{E} h(t, p) g(p) d p
$$

Recall that we assume in this paper that $K$ and $L$ are injective, so that $K^{-1}$ and $L^{-1}$ exist. Let us state a simple lemma.

Lemma 2.1. One has

$$
[L F, L G]=(F, G)_{H_{0}},
$$

provided that $R K H S H_{K}$ is defined by the kernel

$$
K(p, q):=\int_{T} \overline{h(t, p)} h(t, q) d m(t) .
$$

Proof. One has

$$
[L F, L G]=\left(K^{-1} L F, L G\right)_{L^{2}(E)}=\left(L^{*} K^{-1} L F, G\right)_{H_{0}}=(F, G)_{H_{0}},
$$

where the operator $L$ in (2.6) is an operator from $H_{0}$ into $H$. The last step in (2.6) is based on the relation:

$$
L^{*} K^{-1} L=I_{H_{0}}
$$

and $I_{H_{0}}$ is the identity operator in $H_{0}$. Let us assume that $L^{-1}$ is a closed, possibly unbounded, densely defined operator from $R(L) \subset H$ into $H_{0}$, where $R(L)$ is the range of $L$. Then formula (2.7) is equivalent to the relation:

$$
K=L L^{*} .
$$

Indeed, in this case one has:

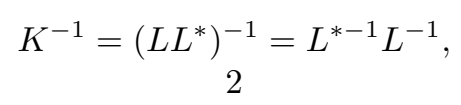


so that (2.7) and (2.8) are equivalent.

Note that under our assumptions about $L^{-1}$ the operator $\left(L^{*}\right)^{-1}$ does exist and $\left(L^{*}\right)^{-1}=\left(L^{-1}\right)^{*}$.

Let us prove that (2.5) is equivalent to (2.8) and, consequently, to (2.7). Using (2.1) and (2.3), one gets:

$$
L L^{*} g=\int_{T} \overline{h(t, p)} \int_{E} h(t, q) g(q) d q d m(t)=\int_{E} K(p, q) g(p) d p
$$

where $K(p, q)$ is defined by (2.5). Since $g(p)$ in (2.10) is arbitrary, this formula implies (2.8), as claimed. Therefore (2.5) implies (2.8), and, consequently, (2.7), and (2.7) implies (2.4) according to (2.6). Lemma 2.1 is proved.

In [Sa1] it is proposed to characterize the range $R(L)$ of linear map (2.1) as the RKHS with the reproducing kernel (2.5). It follows from Lemma 2.1 that if one puts the inner product (1.1) of $H_{K}$, with $K(p, q)$ defined in (2.5), on the set $R(L)$, then $L: H_{0} \rightarrow H_{K}$ is an isometry (see (2.6)), and $R(L)=H_{K}$.

In general one cannot describe the norm in $H_{K}$ in terms of some standard norms, such as the Sobolev norm.

Therefore the above observation (that $R(L)=H_{K}$ if one puts the norm of $H_{K}$ onto $R(L)$ ) does not solve the problem of characterization of the range of $L: H_{0} \rightarrow H$.

This point was discussed in [R2]. On the other hand, some cases are known when one can characterize the norm in $H_{K}$ in terms of the Sobolev norms (positive or negative) [R1].

It is also claimed in $[\mathrm{Sa} 1,2]$ that an inversion formula exists for a general linear transform (2.1) ([Sa2, p.56, formula (31)]).

This inversion formula is derived under the assumption [Sa2, p.58] that $H_{K}$ is the space $L^{2}(E, d \mu)$, where $d \mu$ is some positive measure.

This assumption means that the kernel $A(p, q)$ of the operator $K^{-1}$ is a distribution of the form $\delta(p-q) w(p)$, where $w(p)$ is the density of the measure $d \mu(p)$, that is $d \mu(p)=w(p) d p$, and $\delta(p-q)$ is the delta function in $L^{2}(E)$, that is, the kernel of the identity operator in $L^{2}(E)$.

This, and the definition of the inverse operator, namely $K K^{-1}=I$, written in terms of kernels, imply:

$$
\delta(p-q)=\int_{E} \delta(p-s) K(s, q) d \mu(s)=K(p, q) w(p),
$$

where we have assumed that $w(p)>0$ is a smooth function, with $v(p):=\frac{1}{w(p)}>0$. Thus, (2.11) implies that the reproducing kernel $K(p, q)$ must be of the form:

$$
K(p, q)=v(p) \delta(p-q)
$$

if one assumes that the inner product in $H_{K}$ is identical to the one in $L^{2}(E, d \mu)$, as indeed S.Saitoh assumes in $[\mathrm{Sa} 2, \mathrm{p} .56]$ and in [Sa1].

Claim: Assumption (2.12) is not satisfied in general, and is essentially equivalent to the formula $L^{-1}=L^{*}$, where $L$ now is considered as an operator from $H_{0}$ into $H_{K}$.

Let us prove the above claim. If $L$ is considered as operator from $H_{0}$ into $H_{K}$, then formula (2.4) implies

$$
L^{*} L=I_{H_{0}}, \quad L: H_{0} \rightarrow H_{K}
$$

and formula (2.6) takes the form:

$$
\|L F\|_{H_{K}}=\|F\|_{0} .
$$

Thus $L: H_{0} \rightarrow H_{K}$ is an isometry (see (2.14)) and $L^{*}$ is the left inverse of $L$ (see (2.13)).

We assume that $L$ is injective, that is, the null-space of the operator $L$ is trivial: $N(L)=\{0\}$. Since, by definition, $H_{K}$ consists of the elements of $R(L)$, that is, $R(L)=H_{K}$, and $L^{*}$ is injective on $R(L)$ by (2.13), it follows that

$$
L^{*}=L^{-1},
$$

where $L^{-1}: H_{K} \rightarrow H_{0}$ is a bounded linear operator. The claim is proved.

Formula (2.15) is equivalent to the inversion formula (31) in [Sa2, p.56], while (2.14) is equivalent to formula (33) in [Sa2, p.57]. 
It is now clear that the assumptions in [Sa1,2] are equivalent to the assumption that $L: H_{0} \rightarrow H_{K}$ is a unitary operator, so that its inverse is $L^{*}$.

This assumption makes the description of the range of $L$ and the inversion formula trivial.

It is suggested in [Sa1] and in [Sa2] to use the norm $\|f\|_{H_{K}}=\left\|L^{-1} f\right\|_{0}=\|F\|_{0}$ on $R(L)$, where $L$ is an injective linear operator, and it was claimed in these works that one gets in such a way a characterization of the range of the operator $L$ defined by formula (2.1). In fact this suggestion does not give a nontrivial and practically useful characterization of the range $R(L)$ of this linear integral operator because the norm $\left\|L^{-1} f\right\|_{0}$ cannot, in general, be described in terms of the usual norms, such as Sobolev or Hölder norms, for example. Likewise, the fact that the inverse of a unitary operator $L$ is $L^{*}$ does not give a nontrivial inversion formula, since the main difficulty is to characterize the space $H_{K}$ in terms of the usual norms (such as Sobolev norms, for example) and to check that $L: H_{0} \rightarrow H_{K}$ is a unitary operator.

Finally, note that if the assumption in [Sa1, p.7] and [Sa2, p.56] holds (this assumption says that $H_{K}$ has the inner product identical to the one of $\left.L^{2}(E, d \mu)\right)$ :

$$
\int_{E} \int_{E} A(p, q) f(p) \overline{g(q)} d p d q=\int_{E} f(p) \overline{g(p)} w(p) d p, \quad \forall f, g \in H_{K}
$$

where $A(p, q)$ is a nonnegative-definite kernel of the operator $K^{-1}$ (see formula (1.1)), and $w(p)$ is a continuous weight function, $0<c_{0} \leq w(p) \leq c_{1}, p \in E$, then

$$
A(p, q)=w(p) \delta(p-q),
$$

which is a relation similar to $(2.12)$.

This means that the assumption in $[\mathrm{Sa} 2, \mathrm{p} .56]$ that the RKHS $H_{K}$ is realizable as $L^{2}(E, d \mu)$ is equivalent to the assumption that the reproducing kernel $K(p, q)$ is of the form (2.12). 


\section{REFERENCES}

[A] N. Aronszajn, Theory of reproducing kernels, Trans. Amer. Math. Soc. 68 (1950), 337-404.

[R1] A.G. Ramm, Random fields estimation theory, Longman, New York, 1990; expanded Russian edition, MIR, Moscow, 1996.

[R2] - On Saitoh's characterization of the range of linear transforms, Inverse problems, tomography and image processing, Plenum Publishers, New York, 1998, pages 125-128; (editor A.G. Ramm)

[Sa1] S. Saitoh, Integral transforms, reproducing kernels and their applications, Pitman Res. Notes, Longman, New York, 1997.

[Sa2] - One approach to some general integral transforms and its applications, Integral transforms and special functions 3 (1995), no. N1, 49-84.

[S] L. Schwartz, Sous-espaces hilbertiens d'espaces vectoriels topologique et noyaux associès, Analyse Math. 13 (1964), 115-256. 